\title{
Epidemiología de la historia de alergia a proteínas de la leche de vaca en niños mayores de un año de edad y su tolerancia
}

\author{
AM. Sacristán Martín ${ }^{\mathrm{a}}$ E. Lanza ${ }^{\mathrm{b}}$, A. Dos Santos Athaide ${ }^{\mathrm{b}}$ \\ aPediatra. CS Pintor Oliva. Palencia. España. \\ ${ }^{b}$ MIR-MFyC. Hospital Río Carrión. Palencia. España.
}

\section{Resumen}

Introducción: la alergia a las proteínas de la leche de vaca (PLV) es una afección frecuente en el primer año de vida, cuya incidencia parece estar aumentando en los países desarrollados.

Objetivos: estudiar, en el ámbito de la Atención Primaria, la prevalencia acumulada de alergia IgE-mediada a las PLV en niños mayores de un año de edad y la adquisición de tolerancia, y factores que pueden estar asociados a ella.

Material y métodos: estudio retrospectivo de las historias clínicas de los pacientes menores de 14 años de un centro de salud en Palencia (2805 niños).

Resultados: el 2,35\% (66) presentó alergia a las PLV. El 57,6\% consiguió tolerar las PLV, la edad media a la que se logró fue de 34,6 meses. No se encontró ningún factor asociado al hecho de alcanzar la tolerancia. Los niños que no presentaban manifestaciones respiratorias de atopia tendían a tolerar antes, pero no de forma estadisticamente significativa.

Conclusiones: se encontró en el ámbito sanitario de la Atención Primaria, una prevalencia elevada de alergia a las PLV IgE-mediada. La probabilidad de alcanzar la tolerancia a las PLV disminuye con la edad y es máxima entre los dos y los tres años. Se necesita realizar un diagnóstico preciso, tanto para el bienestar de los pacientes y sus familias como para evitar gastos sanitarios innecesarios.

Palabras clave: Alergia a proteínas de la leche de vaca. Alergia a alimentos. Epidemiología. Tolerancia. Infancia.

Epidemiology of cow's milk allergy and its tolerance in children more than one year old

Abstract

Introduction: allergy to cow's milk (CM) proteins is a frequent condition in the first year of life and its incidence seems to be increasing in the developed countries.

Objectives: to study the accumulated prevalence of IgE-mediated cow's milk allergy (CMA) in children more than one year old at primary care, and tolerance development and factors that can be associated to it.

Ana M. ${ }^{a}$ Sacristán Martín, joseignacioyana@msn.com

Los autores declaran no presentar conflictos de intereses en relación con la preparación y publicación de este artículo. 
Material and methods: retrospective study of $(2,805)$ clinical histories of patients under 14 years in a primary health centre.

Results: we found cow's milk allergy in $2.35 \%$ (66); $57.6 \%$ were able to tolerate CM at an average age of 34.6 months. We did not find any factors associated to the development of tolerance. Children who did not suffer respiratory symptoms tended to reach tolerance earlier but there was not statistical significance.

Conclusions: we found a high prevalence of IgE-mediated CMA in primary care. The probability to develop tolerance to CM falls with age being the maximum in the second and third years of life. Carrying out an appropriated diagnosis is needed for patient and family comfort and for avoiding unnecessary health care expenditure.

Key words: Cow's milk allergy. Food Allergy. Epidemiology. Tolerance. Infant.

\section{Introducción}

La alergia a las proteínas de la leche de vaca (PLV) es frecuente, comienza en etapas tempranas de la vida, generalmente antes de los seis meses, y parece estar aumentando en los países desarrollados. Entre un 0,29 y un $5,3 \% \%^{1-3}$ de los niños presenta alergia a las PLV, las cifras oscilan en función de la metodología diagnóstica y las edades estudiadas.

En España, los alimentos que producen alergia con más frecuencia en los niños son la leche, el huevo y el pescado ${ }^{4}$. Existen varios mecanismos en la producción de la alergia a las PLV: tipo I o hipersensibilidad inmediata mediada por IgE, tipo II o de reacción citotóxica, tipo III o por complejos inmunes y tipo IV o reacción mediada por células.

\section{Objetivos primarios}

Conocer la prevalencia acumulada de alergia a las PLV IgE-mediada en niños mayores de un año; proporción de niños que alcanzan la tolerancia y edad a la que lo logran.

\section{Objetivos secundarios}

Investigar la presencia de factores predictores de tolerancia.

\section{Material y métodos}

Entre junio y diciembre de 2010 se revisaron las historias clínicas de 2805 niños de uno a 14 años pertenecientes al centro de salud Pintor Oliva en la ciudad de Palencia (España).

Se consideró como probable alergia a las PLV a los niños con clínica sospechosa (lesiones cutáneas del tipo urticaria o angioedema con manifestaciones digestivas o sin ellas). Para confirmar la alergia se realizaron pruebas epicutáneas con extractos de leche entera de vaca, alfa- $y$ betaalbúmina en una concentración de 5 $\mathrm{mg} / \mathrm{ml}$, y caseína $(10 \mathrm{mg} / \mathrm{ml})$; la histamina $(10 \mathrm{mg} / \mathrm{dl})$ se utilizó como control positivo y el suero glucosalino como control 
negativo. La prueba es positiva cuando a los 15 minutos el diámetro del habón es mayor de $3 \mathrm{~mm}$ del control negativo. También se midió la lgE específica en suero para PLV y subclases, mediante enzimoinmunoanálisis fluorométrico (Inmuno $C A{ }^{\circledR} 250$ Phadia), con resultado positivo si era mayor de 0,35 kU/I.

Se consideró que tenían alergia a las PLV IgE-mediada aquellos niños con clínica sugestiva y al menos un resultado positivo en las pruebas epicutáneas, y/o IgE específica en suero, y que mejoraron al retirar la leche de vaca y sus derivados.

Se excluyó a los niños que tenían intolerancia a las PLV no mediada por IgE, cuyos resultados para las pruebas anteriores fueron negativos. No se excluyeron a priori niños que tuvieran algún otro tipo de enfermedad digestiva.

Se realizó un estudio descriptivo mediante el programa estadístico G-Stat $2.0^{\circledR}$, las variables cualitativas se expresaron como porcentajes y test de chi cuadrado, las variables cuantitativas se estudiaron mediante el test de la $t$ de Student. Se hizo regresión logística para el estudio multifactorial.

\section{Resultados}

De los 2805 niños menores de 14 años estudiados 66 (2,35\%) fueron alérgicos a la PLV (mediada por IgE). El 42,4\% eran niñas y el $57,6 \%$ varones, con una edad media de 6,97 años ( $\pm 3,9$; rango: 1,2-13,8 años) y la siguiente distribución por edad fue: menores de tres años, el $16,9 \%$; de tres a seis años, el 33,9\%,y siete o más años, el 49,2\%.

En cuanto al tipo de parto, el 78,5\% procedía de parto eutócico, el $23 \%$ de cesárea y el 1,92\% de parto instrumental.

Con respecto al tipo de lactancia, el $90,2 \%$ inició lactancia materna exclusiva; el 7,85\%, mixta desde el nacimiento, y solo el 1,96\%, artificial desde el inicio.

Presentaba antecedentes familiares de enfermedades atópicas el 34,43\%; de ellos, el familiar más frecuentemente afectado fue el padre $(40,9 \%)$, en el $27,3 \%$ la madre, los hermanos en el $22,7 \%$ y ambos progenitores en el $9,1 \%$. El 28,6\% padecía más de un tipo de manifestación atópica, un 23,8\% rinoconjuntivitis y otro $23,8 \%$ dermatitis atópica, asma el 19,5\% y alergia alimentaria como única manifestación de la atopia solo ocurrió en un familiar $(4,7 \%)$.

El $46,1 \%$ de los alérgicos a la PLV (30 de 66) tenía otra manifestación atópica: el 30\%, asma; el 23,3\%, dermatitis atópica; el $10 \%$, rinoconjuntivitis polínica, y el $36,7 \%$, dos o más manifestaciones. El $34,8 \%$ tenía procesos respiratorios (rinoconjuntivitis alérgica y/o asma), además 
de la alergia a las PLV. Ninguno padecía otra enfermedad digestiva o inmunológica. En la tabla 1 se reflejan las características de los niños con alergia a las PLV.

La edad media al diagnóstico fue 6,9 meses ( $\pm 5,79$; rango: 1-36 meses), el $95 \%$ durante el primer año de vida. Excluyendo a los tres pacientes que empezaron la alergia a las PLV entre los 24 y los 33 meses, la edad media al diagnóstico fue 5,8 meses ( $\pm 2,3$ meses).
Se realizaron pruebas alérgicas a neumoalérgenos a 16 pacientes mayores de seis años con manifestaciones respiratorias: fueron positivas en $13(81,25 \%)$, y el polen de gramíneas, de forma aislada o asociado a otros, fue el alérgeno implicado con más frecuencia $(61,25 \%)$.

El $30,8 \%$ presentaba alergia a otros alimentos: el 85,2\%, al huevo; el 16,9\%, a dos alimentos, y un $13,8 \%$, a más de dos.

\begin{tabular}{ll}
\hline Tabla 1. Descripción de la muestra: 66 niños con alergia a las proteínas de leche de vaca \\
\hline Variables & Porcentajes y medias \\
\hline Sexo & $42,4 \%$ niñas \\
\cline { 2 - 2 } & $57,6 \%$ varones \\
\hline Edad media & 6,9 años $\pm 3,9$ \\
\hline Tipo de parto & $78,5 \%$ eutócico \\
\cline { 2 - 2 } & $23 \%$ cesárea \\
\cline { 2 - 2 } & $1,9 \%$ instrumental \\
\hline Lactancia al nacimiento & $90,2 \%$ exclusiva \\
\cline { 2 - 2 } & $7,8 \%$ mixta \\
\hline Antecedentes familiares de atopia & $1,9 \%$ artificial \\
\hline Otras manifestaciones atópicas & $34,4 \%$ \\
\hline Edad media al diagnóstico & $46,1 \%$ \\
\hline Alergia a otros alimentos & 5,8 meses $\pm 2,3 *$ \\
\hline Consiguieron tolerar las PLV & $30,8 \% * *$ \\
\hline Edad media en la que se consiguió la tolerancia & $57,6 \%$ \\
\hline *Ex proteínas de leche de vaca. & 34,6 meses $\pm 21,6$ \\
\hline
\end{tabular}


Con el tiempo, el 57,6\% consiguió tolerar las PLV, a una edad media de 34,6 meses $( \pm 21,61)$, con edades entre los nueve y los 84 meses; la mayoría lo logró antes de los tres años $(69,7 \%)$. En la figura 1 se refleja la persistencia de la alergia a las PLV en función de la edad.

$\mathrm{Ni}$ en el análisis de regresión logística, ni al estudiar cada factor por separado, se encontró ningún factor asociado a la consecución de la tolerancia. En la tabla 2 se reflejan los factores estudiados en relación con alcanzar o no la tolerancia a las PLV.

Los niños que no padecían manifestaciones respiratorias de la atopia tendían a alcanzar la tolerancia antes (el 78,26\% antes de los tres años) frente a los que sí la padecían $(46,7 \%)$, pero no fue significativa (odds ratio [OR]: 4,1; intervalo de confianza del 95\% [IC 95\%]: 0,99 a 16,98). Tampoco se encontró relación entre tener antecedentes familiares de atopia y alcanzar la tolerancia a los tres años o más (OR: 3,3; IC 95\%: 0,7 a $13,8)$.

\section{Discusión}

La Academia Europea de Alergia e Inmunología Clínica ${ }^{5}$ define la hipersensibilidad a alimentos como una reacción adversa que causa síntomas reproduci-

Figura 1. Persistencia de alergia a las proteínas de leche de vaca (en porcentajes) según la edad.

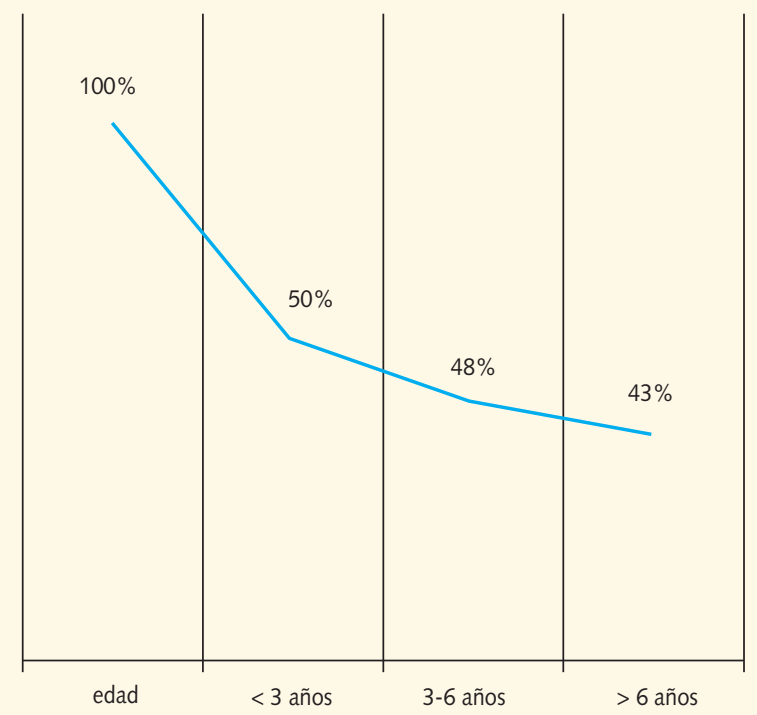




\begin{tabular}{lll}
\hline \multicolumn{4}{l}{ Tabla 2. Factores de riesgo y posibilidad de alcanzar la tolerancia a las proteínas de leche de vaca } \\
\hline Factores de riesgo & OR (IC 95\%) & Probabilidad \\
\hline Parto eutócico & $1,3(0,4$ a 4,2) & $\mathrm{P}=0,64$ \\
\hline Tipo de lactancia inicial exclusiva & $6,5(0,6$ a 63,9$)$ & - \\
\hline Antecedentes familiares de atopia & $1,8(0,2$ a 16,0$)$ & $\mathrm{P}=0,58$ \\
\hline Antecedentes personales de atopia & $1,1(0,36$ a 3,4$)$ & $\mathrm{P}=0,94$ \\
\hline Tener otras alergia alimentarias & $0,7(0,23$ a 2,04$)$ & $\mathrm{P}=0,5$ \\
\hline Tener sensibilidad a neumoalergenos & $0,2(0,0$ a 5,1$)$ & $\mathrm{P}=0,19$ \\
\hline IC: intervalo de confianza; OR: odds ratio. & & \\
\hline
\end{tabular}

bles objetivamente tras la exposición a un alimento en dosis toleradas por sujetos normales. Las PLV son las primeras proteínas a las que se enfrentan los niños y su alergia constituye la forma de alergia alimentaria más frecuente en los primeros meses de vida, su inicio después de los dos años es excepcional.

La alergia a las PLV es un importante problema de salud y un motivo de preocupación continua de los padres, por la necesidad de seleccionar alimentos adecuados exentos de PLV, no siempre bien etiquetados. Quienes la padecen tienen mayor riesgo de presentar asma alérgica lo largo de la vida y sensibilización a múltiples antígenos, tanto alimentarios como respiratorios, y la posibilidad de presentar síntomas de gravedad, llegando incluso a poner en riesgo su vida.

Existe controversia sobre el posible au- mento de la alergia a alimentos ${ }^{6,7}$. La prevalencia real de la alergia a las PLV en la infancia es difícil de precisar, debido, entre otros factores, a la falta de uniformidad de los estudios y a los distintos mecanismos implicados ${ }^{1-3}$. En este momento existe una gran confusión terminológica y es frecuente utilizar en la práctica diaria, e incluso en la literatura, términos semejantes (intolerancia) para referirse a entidades diferentes ${ }^{4}$.

La alergia a las PLV mediada por IgE es la forma mejor caracterizada por existir pruebas de laboratorio que hacen que el diagnóstico sea más preciso. Siempre que se observe mejoría con la retirada de las PLV no es necesaria la prueba de provocación para el diagnóstico inicial ${ }^{8}$.

Los niños con alergia a las PLV de este estudio fueron principalmente alimentados al pecho de forma exclusiva, incluso con mayor frecuencia que la población 
general de Castilla y León y los declarados por nuestro hospital de referencia (el $82,7$ y el $72 \%$, respectivamente) $)^{9,10}$, dato que concuerda con otros estudios sobre alergia a las PLV en los que la mayoría de los pacientes habían sido alimentados al pecho de forma exclusiva ${ }^{8}$.

La prevalencia encontrada es superior a la de otros estudios ${ }^{1,2}$, pero en rangos admitidos por la bibliografía ${ }^{4}$. La elevada prevalencia puede estar relacionada con la frecuencia con que se administran derivados de PLV en los primeros días de vida $(73 \%)$, en la maternidad de nuestro hospital de referencia ${ }^{10}$. Se considera que la toma intermitente de pequeñas cantidades de PLV durante la lactancia materna es un factor desencadenante de la alergia IgE-mediada, ya que imposibilita la adquisición de tolerancia propiciada por la administración continuada, considerándose las pequeñas tomas ofrecidas ocasionalmente en la maternidad o en los primeros días de vida como factores de riesgo para la aparición de la alergia a las PLV.

El 30,8\% presenta alergia a otros alimentos, el 16,9\% tenía alergia a dos alimentos y un $13,8 \%$ a más de dos. Esto ocasiona grandes restricciones dietéticas y dificultad para su alimentación, y conduce a que estos niños no puedan integrarse con normalidad en distintas actividades, como comedores escolares, campamentos, etc.

De los niños con manifestaciones respiratorias de atopia, el 81,25\% estaba sensibilizado a neumoalérgenos, característica de la marcha atópica.

La edad media al diagnóstico fue algo mayor que la descrita por Martorell, pero el $95 \%$ se diagnosticó en el primer año de vida ${ }^{8}$.

El factor de riesgo principal para presentar alergia a las PLV es la carga atópica familiar. En nuestro estudio, la presencia de antecedentes familiares de atopia fue inferior a la reflejada en otros ${ }^{8,11}$.

El porcentaje de pacientes que presentaba más de una manifestación atópica fue similar a otros estudios ${ }^{12}$.

El porcentaje que alcanzó tolerancia fue similar al encontrado por $\operatorname{Dias}^{13}$ en Portugal a los diez años, aunque en sus estudios no todos presentan alergia IgEmediada, lo mismo ocurría con la muestra del estudio de Tikkanen ${ }^{12}$.

En nuestro estudio existe una tendencia a que los niños con manifestaciones respiratorias de atopia (asma y/o rinoconjuntivitis) alcancen la tolerancia más tarde. En el estudio de Santos ${ }^{11}$, los niños con alergia IgE-mediada o alergia a múltiples alimentos, si alcanzaban la tolerancia lo hacían siempre después de los dos años. Parece que la atopia en sí misma es 
un factor de riesgo para la prolongación de la alergia a las PLV, mientras que los niños que presentan intolerancia a las PLV parecen recuperarse antes.

Haciendo una estimación de las necesidades medias al mes de hidrolizados en los menores de dos años, encontramos un gasto para el sistema de salud de $321,9 €$ por niño menor de dos años. Es necesario realizar un correcto diagnóstico y actuar sobre el único factor asociado a la aparición de la alergia a las PLV que resulta modificable: la administración de pequeñas cantidades de PLV en los primeros días de vida en niños que son amantados de forma natural.

\section{Conclusiones}

Encontramos una elevada prevalencia de alergia a las PLV IgE-mediada, que debe hacernos reflexionar sobre los factores asociados, sobre todo en aquellos en los que podemos intervenir; la probabilidad de alcanzar la tolerancia a las PLV disminuye con la edad, siendo máxima de los dos a los tres años de vida; después, la curva de persistencia de la alergia disminuye lentamente. Se necesita hacer un diagnóstico preciso, tanto para bienestar de los pacientes y sus familias, como para evitar gastos sanitarios innecesarios.

\section{Bibliografía}

1. Kucukosmanoglu E, Yazi D, Yesil O, Akkoc T, Gezer M, Ozdemir C et al. Prevalence of immediate hypersensitivity reactions to cow's milk in infants based on skin prick test and questionnaire. Clin Exp Allergy. 2009;39(7):1027-35.

2. Sanz Ortega J, Martorell A, Michavilla A, Nieto A y Grupo de Trabajo para Alergia Alimentaria. Estudio de la incidencia de alergia mediada por lgE frente a la proteína de leche de vaca en el primer año de vida. An Esp Pediatr. 2001;53:536-9.

3. Frank Kneepkens CM, Meijer Y. Clinical practice. Diagnosis and treatment of cow's milk allergy. Eur J Pediatr. 2009;168:891-6.

4. Rona RJ, Keil T, Summers C. The prevalence of food allergy: a meta-analysis. J Allergy Clin Immunol. 2007:120:638-46.
5. Johansson SGO, Hourricane JOB, Bousquet J, Bruiijnzeel-Koomen C, Dreborg S, Haahtela T et al. Position paper. A revised nomenclature for allergy. Allergy. 2001;56:813-24.

6. Venter C, Pereira B, Voigt K. Prevalence and cumulative incidence of food hypersensitivity in the first 3 years of life. Allergy. 2008;63:354-9.

7. Hu Y, Chen J, Li H. Comparison of food allergy prevalence among Chinese infants in Chongqing, 2009 versus 1999. Pediatr Int. 2010;52(5): 820-4.

8. Martorell A, Plaza AM, Boné J, Nevotd S, García Ara MC, Echeverria L et al. Cow's milk protein allergy. A multi-centre study: clinical and epidemiological aspects. Allergol Immunopathol. 2006;34(2):46-53.

9. Sacristán Martín AM, Lozano Alonso JE, Gil Costa M, Vega Alonso AT, Red Centinela Sanitaria 
de Castilla y León. Situación actual y factores que condicionan la lactancia materna en Castilla y León. Rev Pediatr Aten Primaria. 2011;13:3346.

10. Bertholt ML, Maldonado Ruiz EM, de la Cruz González Torroglosa M, Peña Valenceja A, Nieto Pastor B, Martin Díaz C. Situación actual de la lactancia materna en el área de salud de Palencia. Encuesta para madres. $60 .^{\circ}$ Congreso de la AEP. Valladolid, 16-18 julio 2011.
11. Santos A, Dias A, Pinheiro JA. Predictive factors for the persistence of cow's milk allergy. Pediatr Allergy Immunol. 2010:21:1127-34.

12. Tikkanen $\mathrm{S}$, Kokkonen J, Juntti $\mathrm{H}$, Aniinima K. Status Of children with cow's milk allergy in infancy by 10 years of age. Acta Paediatr. 2000;89:1174-80.

13. Dias A, Santos A, Pinheiro JA. Persistence o cow's milk allergy beyond two years of age. Allergol Immunopathol (Madr). 2010;38(1):8-12. 
